\title{
Use of type VII collagen gene (COL7A1) markers in prenatal diagnosis of recessive dystrophic epidermolysis bullosa
}

M G S Dunnill, C H Rodeck, A J Richards, D Atherton, B D Lake, M Petrou, R A J Eady, F M Pope

\begin{abstract}
Generalised recessive dystrophic epidermolysis bullosa (EB) is a severe inherited disease in which patients suffer from blistering and scarring of the skin and mucous membranes after minor mechanical trauma. Tight genetic linkage has been established to the type VII collagen gene (COL7A1) at 3p21, with no evidence of locus heterogeneity. Several COL7A1 mutations have now been identified in recessive dystrophic EB patients. Prenatal diagnosis has been performed by examination of a fetal skin biopsy taken at about 16 weeks' gestation, and relies on identification of characteristic ultrastructural and immunohistochemical changes. We have now achieved a first trimester prenatal diagnosis using intragenic and flanking COL7A1 markers in a pregnancy at risk for recessive dystrophic EB. Segregation of the informative markers predicted the baby would be an unaffected carrier. The pregnancy continued to term and a healthy baby was born, confirming this result.
\end{abstract}

(f Med Genet 1995;32:749-750)

Recessive dystrophic epidermolysis bullosa is an inherited disorder of skin and mucous membranes characterised by blistering and scarring after minor mechanical trauma. Mutilating deformities and squamous cell carcinoma may occur, and patients frequently die in the third or fourth decade. Electron microscopy has shown abnormalities of anchoring fibrils, attachment structures composed of type VII collagen. ${ }^{1}$ Genetic linkage studies have implicated the type VII collagen gene (COL7A1) at $3 \mathrm{p} 21$. The combined maximum lod score ( $\mathrm{Zmax}$ ) from studies of generalised recessive dystrophic EB using an intragenic PvuII restriction fragment length polymorphism (RFLP) is 7.48 at a recombination fraction $(\theta)$ of zero. ${ }^{23}$ None of the families showed any evidence of recombination, firmly supporting locus homogeneity for this disease. Pathogenic mutations on COL7Al have now been identitfied, confirming the role of COL7A1 in this disease. ${ }^{4-7}$

Prenatal diagnosis is often requested by parents of children with severe recessive dystrophic EB. Fetal skin biopsy allows prenatal diagnosis at 16 weeks' gestation. ${ }^{8}$ Although this is an accurate and reliable method, the necessity of a late midtrimester termination is disadvantageous. We have recently achieved first trimester prenatal diagnosis in a pregnancy at risk for severe recessive dystrophic EB using intragenic and flanking COL7A1 markers.

The consanguineous parents of a child with generalised recessive dystrophic EB requested prenatal diagnosis in the 10th week of a subsequent pregnancy. A chorionic villus sample was obtained at $10 \frac{1}{2}$ weeks' gestation using transcervical biopsy forceps and DNA was extracted using standard methods after elimination of maternal tissue contamination. The polymerase chain reaction (PCR) was used to amplify DNA fragments containing the markers, and PCR products were then digested with the appropriate restriction enzymes. ${ }^{39}$ The PvuII polymorphism, which lies within the NC-1 coding domain of COL7A $1,{ }^{2}$ was fully informative, and predicted that the fetus would be an unaffected heterozygous carrier (figure A). A second polymorphic MspI site flanking COL7A1 (D3S2) was partially informative, and indicated fetal inheritance of the maternal allele carrying the mutation (figure $\mathrm{B}$ ). The absence of the $(-)$ allele from the fetal sample helped to exclude maternal contamination. The pregnancy was continued to term and a healthy baby was born, confirming the result.

The gene encoding type VII collagen is highly complex with 118 exons and $9 \cdot 2 \mathrm{~kb}$ cDNA. ${ }^{10}$ Furthermore the mutations so far described have been largely unique to individual families and do not cluster. ${ }^{47}$ Without common mutations and in the context of a large and complex gene, it may not be feasible to identify the pathogenic mutations in all families requesting prenatal diagnosis. Appropriate polymorphic markers within and tightly flanking COL7A1 will therefore be important for prenatal diagnosis. The speed and accuracy of DNA based testing coupled with the ability to obtain fetal 
A

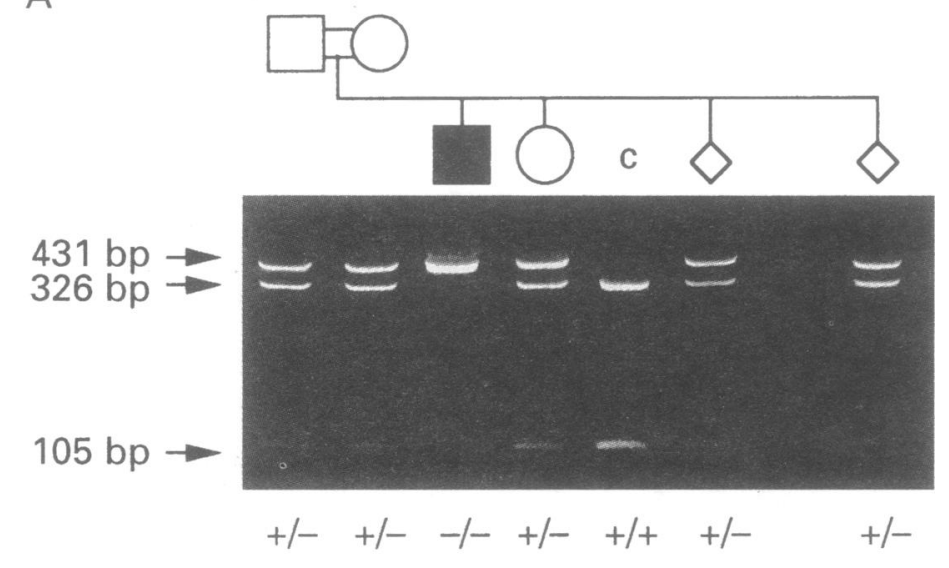

B

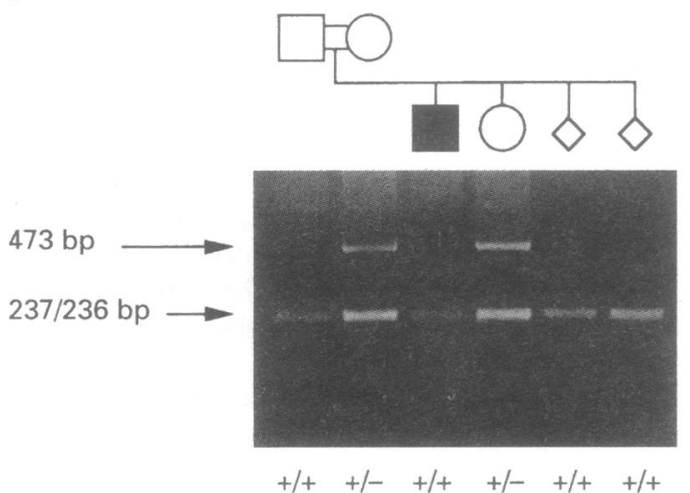

Marker results. Open box, unaffected male; closed box, affected male; open circle, unaffected female; open diamond, fetal sample. $(A)$ The PvuII marker. The affected child is homozygous for the (-) allele. The fetal sample was run in duplicate and is heterozygous (+l-), indicating unaffected carrier status. A control sample, $C$, known to be homozygous $(+1+)$ was also run in the 5th lane. (B) The D3S2 flanking marker. The mother is heterozygous $(+1-$ ) and the affected child is homozygous $(+1+)$, indicating segregation of the maternal mutation with the $(+)$ allele. The fetus is homozygous $(+1+)$ showing inheritance of the maternal mutation.

DNA from chorionic villus in the first trimester mean that this method will supersede fetal skin biopsy in the future.

\section{Note added in proof}

After we submitted our manuscript for publication, Hovnanian et $a l^{11}$ published a report on prenatal diagnosis of generalised recessive dystrophic EB based on genotype analysis with COL7A1 polymorphic markers or direct mutation detection.

We would like to acknowledge Miss J Denyer for her help in counselling the family and Dr Alain Hovnanian for his kind advice. We thank DEBRA UK and the MRC for partial funding of laboratory work. MGSD is supported by a DEBRA research fellowship.

1 McGrath JA, Ishada-Yamamoto A, O'Grady A, Leigh IM, Eady RAJ. Structural variations in anchoring fibrils in dystrophic epidermolysis bullosa: correlation with type VII collagen expression. $\mathcal{F}$ Invest Dermatol 1993;100:366-72.

collagen expression. F Invest Dermatol 1993;100:366-72.
2 Hovnanian A, Duquesnoy P, Blanchet-Bardon C, et al. Genetic linkage of recessive dystrophic epidermolysis bulGenetic linkage of recessive dystrophic epidermolysis bul-
losa to the type VII collagen gene. $\mathcal{f}$ Clin Invest 1992;90: losa to the
$1032-6$.

3 Dunnill MGS, Richards AJ, Milana G, et al. Genetic linkage to the type VII collagen gene (COL7A1) in 26 families with generalised recessive dystrophic epidermolysis bullosa and anchoring fibril abnormalities. $\mathcal{F}$ Med Genet 1994;31: 745-8.

4 Hovnanian A, Hilal L, Blanchet-Bardon C, et al. Recurrent nonsense mutations within the type VII collagen gene in patients with severe recessive dystrophic epidermolysis bullosa. Am 7 Hum Genet 1994;55:289-96.

5 Hilal L, Rochat A, Duquesnoy P, et al. A homozygous insertion-deletion in the type VII collagen gene (COL7A 1 ) in Hallopeau-Siemens dystrophic epidermolysis bullosa. Nature Genet 1993;5:287-93.

6 Christiano AM, Anhalt G, Gibbons, S, Bauer EA, Uitto J. Premature termination codons in the type VII collagen gene (COL7A1) underlie severe, mutilating recessive dygene (COL7A1) underlie severe, mutilating recessive dy-
strophic epidermolysis bullosa. Genomics 1994;21:160-8.

7 Dunnill MGS, Richards AJ, Milana G, Mollica F, Eady RAJ, Pope FM. A novel homozygous point mutation in the collagen VII gene (COL7A1) in two cousins with recessive dystrophic epidermolysis bullosa. Hum Mol Genet 1994;3:1693-4.

8 Rodeck CH, Easy RAJ, Gosden C. Prenatal diagnosis of epidermolysis bullosa letalis. Lancet 1980;i:949-52.

9 Ganley P, Rabbits PH. Polymerase chain reaction (PCR) for detection of Msp I polymorphism at the D3S2 locus. Nucleic Acids Res 1991;19:3761

10 Christiano AM, Greenspan DS, Lee S, Uitto J. Cloning of human type VII collagen. Complete primary sequence of the $\alpha 1$ (VII) chain and identification of intragenic polymorphisms. F Biol Chem 1994;269:20256-62.

11 Hovnanian A, Hilal L, Blanchet-Bardon C, et al. DNAbased prenatal diagnosis of generalized recessive dystrophic epidermolysis bullosa in six pregnancies at risk trophic epidermolysis bullosa in six pregnancies a
for recurrence. $\mathcal{F}$ Invest Dermatol 1995;104:456-61. 